\title{
LXIV. A list of about five hundred collieries in and near to Derbyshire
}

\section{Mr. John Farey Mineralogical Surveyor}

To cite this article: Mr. John Farey Mineralogical Surveyor (1810) LXIV. A list of about five hundred collieries in and near to Derbyshire, Philosophical Magazine Series 1, 35:146, 431-438, DOI: $10.1080 / 14786441008563119$

To link to this article: http://dx.doi.org/10.1080/14786441008563119

曲 Published online: 18 May 2009.

Submit your article to this journal $\sqsubset \pi$

Џ Article views: 5

Q View related articles $\square$ 
E.s.d.

$2 \quad 3 \quad 6$ Accumulating as above for 50 years, will amount to

Add the value of the tree to be cut down at the end of 200 years Total amount in 200 years -
And carrying forward this calcu-
lation, the total amount of the produce in 300 years would amount to
\&. s. $d$.

$24 \quad 0 \quad 0$

236

In former times, when the value of oak-woods was estimated by the number of hogs their acorns would fatten, the great age of trees would be of small consideration; but in the present times, I am persuaded, that if gentlemen who have many trees standing of the age of 150 years and upwards, would give this subject its due consideration, they will be aware of the immense loss to which they are voluntarily subjecting themselves.-And this great loss is much to be regretted, in a political point of view, especially as the produce of this island is insufficient for its necessary consumption.

My motives for troubling you with this hasty production. are, to promote the good of the public, by endeavouring to persuade gentlemen to bring forward well ascertained facts, respecting the most profitable management of growing timber trees, and to induce them to investigate, with accuracy, this very curious and important subject. If you think it is likely to have those effects, I shall thank you to lay this paper before the Society of Arts, \&c. for their consideration.

No. 99, High Holbcrn,

I am, sir, your obedient servant, Oct. 1809 .

Charles Waistell.

LXIV. A List of about Five Hundred Collieries in and near to Derliyshire. By Mr. John E AREY, Mineralogical Surveyor.

To Mr. Tillocil.

SIR, I Incrosen a list, of such collieries as I have either visited or obtained information concerning, in the course of my recent examination of the county of Derby, and the borders of the seven adjacent counties. Should you deem the 
the same worthy of a place in your Philosophical and Geological Magazine, it may be proper to observe, that the names of the places in the first column, arc not always those of the parish in which the coal-works are situate, but of the nearest or most convenient place, shown in greneral maps, by which to refer to the precise site of the collieries. Also, that many of these works are now discontinued : yet as in almost every instance, more of this valuable mineral remains still ungotten, in or near to the same spot, the recording of all places where coals have at any time been worked, seems an object of some importance. In the report on the county of Derby, which I am at present employed in preparing, for the Board of Agriculture, I intend to give in one alphabetical list, the bearings and distances of each of these collieries, from the towns mentioned in the first column, with the particular place in the series of strata to which each colliery is to be referred, as far as $1 \mathrm{am}$ able in the present instance: the collating of the great mass of information which $I$ have been favoured with, from the several coal-masters, and their agents and workmen, being yet unfinished, as well as the reexamination of some parts of the interesting coalfields, within the limits of my intended map, in districts where the alluvial coverings, the faults and stupendous dislocations of the strata, have presented great, but as I trust, not insuperable difficulties, to the complete elucidation of these bighly important strata. In the report I shall distinguisli such of the above collieries as now are, or recently have been, in work. Where no county is mentioned to the places in the first column, Derbyshire is to be understood; in which county I find the coal strata or measures, distributed over not less than 190,000 acres of its surface! I shall esteem it a great favour, if proprietors of estates, or others, who happen to be possessed of correct accounts of the sinkings or measures at any of the collieries below, in documents to which I may not have had access, or respecting any ancient collieries within these limits, which may have eluded my inquiries, that they will communicate copies of such particulars (addressed as below) as soon as convenient; carefully distingishing the bearing and distance from one at least of the above places; the time of ceasing to work, old collieries, if known, \&c. The borings or sinkings, whore trials to any depth have been made, whether successful or not, would be alike acceptable, such being the materials from which, principally, a correct ac- 
count of the subterranean geography of the district, or the knowledge of its strata, is to be drawn.

22, Upper Crown Street, West-

$$
\begin{aligned}
& \text { I am, sir, } \\
& \text { Your obedient servant, } \\
& \text { JoH N FAREY. }
\end{aligned}
$$$$
\text { minster, June } 5,1810 .
$$

Places' Names.

Names of Collieries.

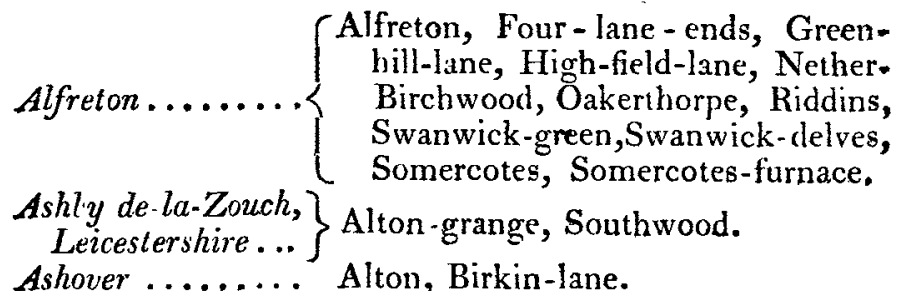
Ashton-under-line, $\{\mathrm{Car}-\mathrm{lane}$, Crickety, Fairbottom, Lancashire .... Hays, Hurst-brook, Knoll. Aston, Yorkshire .. $\begin{aligned} & \text { Bigging, Conduit-moor, Fox-Ears, } \\ & \text { Lawn (or Casteven or Kesteven), } \\ & \text { Pidgeon-bridge, Aston- common, } \\ & \text { Swallow-nest. }\end{aligned}$ Attercliff, Yorks... $\left\{\begin{array}{l}\text { Attercliff - common, Bright - side, } \\ \text { Darnall,High-hazels, Tinsley-park, } \\ \text { Washford. }\end{array}\right.$ Awsworlh, Notts. Awsworth, Newthorpe-common. Parlborough .... $\begin{aligned} & \text { Barlborough - common, Beighton - } \\ & \text { field, Hazlewell, Westfield, Kni- } \\ & \text { taker, Pebley - lane, Spinkhill - } \\ & \text { common. }\end{aligned}$ Barlow........ $\left\{\begin{array}{l}\text { Barlow-common, Cutthorpe, Far- } \\ \text { lane, High-ash, Sudbrook, Wil- } \\ \text { ders-green. }\end{array}\right.$ Barnsley, Yorks... Gober-hall. Baslow......... Baslow, Chatsworth old Park,

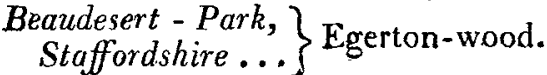
Beeley ......... Beeley-moor, Harwood-Grange. Beighton ....... Berley-moor, Nether-field. Belper ....... $\left\{\begin{array}{l}\text { Belper-gutter, Belper-lane-end, Bel- } \\ \text { per Town, Bent, Chevin-side, } \\ \text { Dally-gutter, Hopping-hill,Open- } \\ \text { wood-gate, Swinney. }\end{array}\right.$ Biddulph, Staff...\{ $\begin{gathered}\text { Biddulph-hall, Crabtree, Childer- } \\ \text { play, Falls, New-Pool, }\end{gathered}$ Vol, 35. No. 146. June 1810. E E Bil. 
Places' Names.

Names of Collieries.

Billorough, Notts. Bilborough, Holly-wood, Nuthall.

Blackfordby, Leices. Blackfordby, Norris-hill.

Blackw'tl ........ Blackwell, Dingmings-dale, Newton.

$\left.\begin{array}{c}\text { Bolderstone-chapel, } \\ \text { Yorkstive ....... }\end{array}\right\}$ Stock-bridge?

Bolsover ....... $\begin{gathered}\text { Palterton, Shutllewood - common, } \\ \text { Stanfrey }\end{gathered}$

Boothorpe, Leices- Little-worth, Milk-hill, Sweet-hill-

tershire....... oak.

$\left.\begin{array}{c}\text { Bradfield-Chupel, } \\ \text { Yorkshire ...... }\end{array}\right\}$ Hagen-field, Holes.

Brimington ..... Brimington, Brimington-moor, Hol-

Brimington ..... \{ lingwood-common, Wildens-mill.

Buxton ......... Goyte-moss, Thatch-marsh.

Calke .......... Brians-coppy.

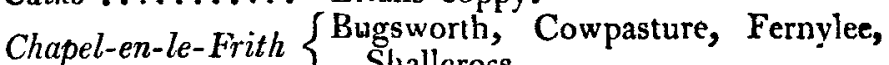
Chapel-Town, $\left\{\begin{array}{c}\text { Shallcross. } \\ \text { Chapel-town, Heasley-Park, Par- }\end{array}\right.$ Yorkshire....... $\left\{\begin{array}{l}\text { kins-wood, Smithy-wood-engine, } \\ \text { Thorncliff. }\end{array}\right.$

Cheadale, Staff... $\left\{\begin{array}{l}\text { Delph-house, East-wall, Eaes, Mov- } \\ \text { berley, Shaw (or Sham), Shaw, } \\ \text { Woodhead. }\end{array}\right.$ Cheddleton, Staff. . \{ Crown-point, Cunsal-wood, Newstead, Shafferlong, Wetley-monr.

Ash-gate, Boythorpe, Chesterfieldtown's-end, Chesterfield-furnace, Calow, Dunston, Grass-hill, Hady,

Chesterfeld ..... \{ $\left\{\begin{array}{l}\text { Hasland, Little-common, Louns- } \\ \text { ley-green, Moor-top, New-Bramp- }\end{array}\right.$ ton, Newbold-common, Newboldfield, Stone-gravel, Tapton, Wdton, Wingerworth-park, Wingerworth-furnace.

Church-Gresley... Gresley, Gresley-hall, Round-hole, Clown ......... Clown.

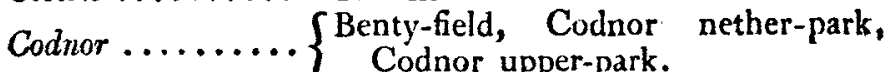
Cole-Orton,Leicest. Cole-Orton, Lount new, Lount old. Conisborough, Yorks. Conisborough, Dennaby.

Cossall, Notts..... Cossall, Robinets.

Crich ........... Plaistow-Green.

Dale-Albea .... \{ $\begin{gathered}\text { Dale-Abbey, Hag, Lower-Hag, } \\ \text { Pingle. }\end{gathered}$ 
Names of Collieries in and near Derbyshire.

Places' Names.

Names of Collieries.

Denly ....... $\left\{\begin{array}{l}\text { Denby, Denby-hall, Roby east-field, } \\ \text { Roby west-field, Smithy-houses. } \\ \text { Dilhorne, Staff. .. }\end{array}\right.$ Disley, Cheshire . $\begin{array}{l}\text { Dilhorne, Parsons - field, Swetley } \\ \text { (Lowes'). }\end{array}$
$\begin{aligned} & \text { Allington, Bank-end, Diglee, Fur- } \\ & \text { nace-clough, Gee, Hag-bank, Hoo- } \\ & \text { lane, Lyme, Lyme-park, Norbury, } \\ & \text { Poynton, Red-acre, Worth. }\end{aligned}$

Dore.......... Dore, Ringing-low-bar.

Dronfield ...... $\left\{\begin{array}{l}\text { Apperknowl-common, Cole-Aston, } \\ \text { Dronfield, Hill-top, Ounston, } \\ \text { Stubley, Woodhouse }\end{array}\right.$

Ducking field, Ches. $\left\{\begin{array}{l}\text { Duckingfield, Dunkirk, Flowery- } \\ \text { feld, Hough-hill, Newton-moor, } \\ \text { Rabbit-hole, Score-wood. }\end{array}\right.$

Duckmanton (long) \{Adelphi-furnace, Duckmanton-com .

Eastwood, Notts. . $\left\{\begin{array}{c}\text { mon, Middle-Duckmanton. } \\ \text { Beggerlee, Brinsley new, Brinsley old, } \\ \text { Eastwood. }\end{array}\right.$

Eccleshall Barlow, 7 Grey - stones, Mill - house, Moss, (in Sheffield, $\}$ Smeling, Trap-lane, WhitleyYorkshire...... $\}$ wood.

Eckington ...... $\begin{aligned} & \text { Arbor-lands, Bole-hill,Bramley-moor, } \\ & \text { Coldwell, Eckington, High-lane, } \\ & \text { Mossborough-moor, Troway. }\end{aligned}$ Flash (in Alston- $\left\{\begin{array}{l}\text { Birchen - booth, Black - clough (or } \\ \text { Beat), Blue-hills, Chest, Dane-head, } \\ \text { Dane-thurn, Diamond-hill, Gold- } \\ \text { sitch, Hazle - barrow, Notbury, } \\ \text { Penny-hole, Whiteshaw. }\end{array}\right.$

Foxton, Staffordsh. \{Clough-head, Foxton-wood, StileFullwood-Chapel, shutt.

(in Sheffield) Yorks. $\}$ Stanage.

Glossop ......... Combs, Simondley.

Greasborough, \} Cinder-hill (or Middle-field), HawYorks......... woud.

Greasley, Notts. .. Beauvale-abby, Greasley, Limes.

Hallyfield, Glcedless-common Hans-

Harthill, Yorks. .. Woodhal!-unor.

Hartshorn ....... Gosley-waste, Hartshorn.

Hathersage ...... Stanage-pole.

$$
\text { Eeq }
$$


Places Names.

Names of Collieries.

Hayfield (in Glossop) $\ldots \ldots \ldots \ldots$.....

Heage ........ \{ Heage, Heage-bent, Morley-park,

Heanor........ Aldercar, Heanor, Langley, Miln-

Heath....... Heath, High-house.

Heather, Leicester. Heather.

Higham ........ Higham.

Hollurook.......... Holbrook.

Holmesfield ...... Bank, Salters-sitch, Thickwood.

Horsley ....... \{ $\begin{aligned} & \text { Horsley, Horsley-woodhouse, Slack- } \\ & \text { fields }\end{aligned}$

Hyde-Chapel, \{ Broomsteer, Denton, Hyde-lane, Cheshire...... W Werneth-low, Woodley.

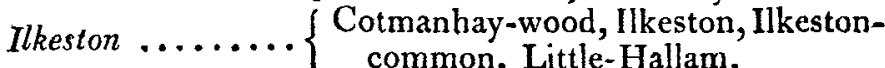
Ipstone, Staff.... $\left\{\begin{array}{l}\text { common, Little-Hallam. } \\ \text { Kowtter, Hay-house, Ipstone, }\end{array}\right.$ Ipstone, Staff.... Knipe, Nether-field.

Killamarsh ..... $\{$ Gander-lane, Killamarsh, Nether-

Kimlerworth, f Blackburn-bank, Bradgate, Kimber-

Yorks. ....... \{ worth, Meadow-hall.

Froghall, Garstone, Hazleș-cross, Kingsley, Staff. .. $\left\{\begin{array}{l}\text { Hodge-hay, Jack-elm, Kingsley- } \\ \text { bank, Lees, Rake-edge, Ross-bank. }\end{array}\right.$ Blake-low, Bollington,- Cliff-bank,

Macclesfield, Ches. Eastborough-lane, Hurdsfield, Macclesfield - common, Riley - clough, Shrigley-fold, Swanco, Throtlesnest.

Marple-Chapel, Ches. Brabins, Chapel-house.

Matlock........ Lea, Lumsdale, Tansley-green. Measham...... $\begin{aligned} & \text { Donisthorpe, Measham, Measham- } \\ & \text { fields, Oakthorpe. }\end{aligned}$

$\{$ Bore-lane, Broadhurst-edge, Comp-

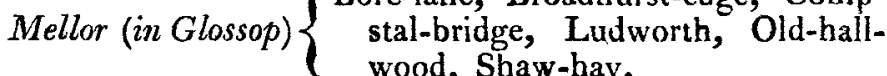
Mexborough, Yorks. Mexborough. Morley .......... Morley. Mossley, Lancash. \{King-bank, Park, Rotches, ScoutMottram, Cheshire. Hague-bank, Hill-end, Hodge-hall. 
Newall (in Stapen- $\left\{\begin{array}{l}\text { Bretby, Pall-fields, Newall, Newall- } \\ \text { park, Perkins, Stanton, Swadlin- } \\ \text { cote, Water-field, Wooden-box, } \\ \text { Wood-field. }\end{array}\right.$

New-Mills (in $\quad$ Eaves-knowl, Lower-house, TorGlossop)...... $\{$ mine, Warps-moor.

Norton, Staff..... Bow-green, Whitfield.

Norton ......... Lees-hall.

North-Winfield.. \{ Ankerbold, Berrisford-moor, Clay-

Oakmoor-Mills (near Alveton) Beelow, Car-wood. Staff..........

Over-Seal, Leicest. Warren-hill-furnace.

Packington, Leicest. Packington.

Pelsall (near Blox- $\{$ Brown-hills, Essington new Colliery, wick) Staff..... Essington-wood, Goscot, Lords-

( hay, Pelsall, Wyrley-bank.

Penistone, Yorks... Bull-house, Flash-house, Fullshaw,

Law, Midhope-stones, Paw-hill.

Pinxton .......... Carter-lane, Pinxton.

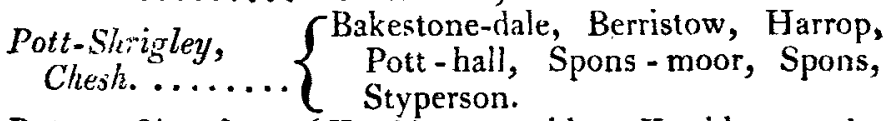

Rainow-Chapel, \{Kerridge-east-side, Kerridge-northChesh.......... end, New-post, Rainow-low.

Rawmarsh, Yorks. SNether-Hough, Over-Hough, Rawmarsh, Stubbing-lane.

Ripley (in Pentrich) \{ Butterly-park, Butterly-car, GreenRipley (in Pentrich) $\{$ wich, Ripley:

Rotherham, Yorks. $\left\{\begin{array}{l}\text { Clough, Herringthorpe, Hill-top, } \\ \text { Kimberworth-park, Mossborough- } \\ \text { common. }\end{array}\right.$ Rudgley, Staff. .. Bruerton.

Crocks-moor, Deep-pits, HarbourSheffeld, Yorks. .. $\begin{aligned} & \text { thorn, Manour,Park-furnace, Ponds, } \\ & \text { Sandy }\end{aligned}$

Shirland........ Shirland, Smithy-moor, Stretton.

Silkstone, Yorks. .. Silkstone.

Skegly, Notts.... Shilo, Dirty-Hucknale, Skegby.

Smalley ....... \{ $\begin{aligned} & \text { Smalley, Smalley-common, Simon- } \\ & \text { field, Woodhouse-lane. }\end{aligned}$ E e 3 
Places' Names.

Names of Collieries.

Smithsby ....... Pistern.

South-Normanton . Berristow, South-Normanton.

Stannington, Yorks. \{ Armitage, Dungworth, Low - ash,

Storrs, Wadsley.

Stanton (by Dale) . Hallam-bridge (or Nutbrook).

$\left.\begin{array}{c}\text { Stanton-Harold, } \\ \text { Leicester. ....... }\end{array}\right\}$ Heath-End, Stanton-Harold.

Slapleford, Notts.. Bramcote.

Staveley ........ Norbrigs; Staveley, Woodthorpe.

Sutton (in Scars- \{Sutton, Sutton-common, Wooddale $). . . \ldots$ nook.

Swepston, Leicester. Swepston.

Tamworth, War-
wickshire ...... Polesworth, Waverton, Wilnecote.

Tankersley, Yorks. Tankersley-park.

Taxhall, Cheshire. Castedge, Gap-sitch.

Temple-Normanton $\left\{\begin{array}{c}\text { Grass-moor, Grass-moor (Platt's), } \\ \text { Lings. }\end{array}\right.$

Teversall, Notts... Dunshill.

Thursfield, Staff. . Bernersley-green.

Ticknall........ Ticknall, White-holly-coppy.

Todwick, Yorks. .. Todwick-moor.

Tilshelf........ Biggin, Harstoft, Tibshelf.

Treton, Yorks..... Cat-cliff.

Wales, Yorks. .... Wales.

Waih, Yorks...... Abry, Wath-wood.

Wentworth-Chapel, f Cortworth, Elsicar, Hoober, Low-

Yorks........ \{ wood, Park-gate, Swallow-woodnook, Wentworth-park.

West-Hallam..... Stanley - conımon, West - Hallam,

West-Hallam windmill-hill.

Whiston, Yorks. .. Royds-moor.

Whittington .... Glass-house-common, Whittington-

Wickersley, Yorks.. Brecks, Hollings-moor.

Wincle-Chapel, \{Green-hill, Hay, Latche, Mouse-trap,

Cheshire...... \{ Robins-clough, Quarnford.

Wirksworth....... Alderwasley, Wigwell.

Wollaton, Notts... Aspley, Trowel-noor, Wollaton.

Woodhead, Chestire Crowden-clough.

Worslorough,Yorks. Stainborough-park, Worsborough.

$\left.\begin{array}{c}\text { Worlley-Chapel, } \\ \text { Yorks........... }\end{array}\right\}$ Deep-car, Hunshelf, Westwood. 